\title{
Changes in Subtypes of Ca Microdomains Following Partial Injury to the Central Nervous System
}

\author{
Ivan Lozića,b, Carole A. Bartlett ${ }^{\mathrm{b}}$, Jeremy A. Shaw ${ }^{c}$, K. Swaminathan Iyer ${ }^{\mathrm{a}}$, Sarah A. Dunlop ${ }^{\mathrm{b}}$, Matt R. \\ Kilburn $^{c}$ and Melinda Fitzgerald ${ }^{b^{*}}$
}

\author{
${ }_{5}$ Received (in $\left.X X X, X X X\right)$ Xth $X X X X X X X X X 20 X X$, Accepted Xth $X X X X X X X X X 20 X X$ \\ DOI: $10.1039 / b 000000 x$
}

Rapid changes in $\mathrm{Ca}^{2+}$ concentration and location in response to injury play key roles in a range of biological systems. However, quantitative analysis of changes in size and distribution of $\mathrm{Ca}^{2+}$ microdomains in specific cell types in whole tissue samples has been limited by analytical resolution and reliance on indirect $\mathrm{Ca}^{2+}$ indicator systems. Here, we combine the unique advantages of nanoscale 10 secondary ion mass spectrometry (NanoSIMS) with immunohistochemistry to directly quantify changes in number, size and intensity of Ca microdomains specific to axonal or glial regions vulnerable to spreading damage following neurotrauma. Furthermore, using NanoSIMS allows separate quantification of Ca microdomains according to their co-localization with areas enriched in P. We rapidly excise and cryopreserve optic nerve segments from adult rat at time points ranging from 5 minutes to 3 months after injury, allowing assessment of Ca microdomains dynamics with minimal disruption due to tissue processing. We demonstrate significantly more non-P 15 co-localized Ca microdomains in glial than axonal regions in normal optic nerve. The density of Ca microdomains not co-localized with areas enriched in $\mathrm{P}$ rapidly, selectively and significantly decreases after injury; densities of Ca microdomains co-localized with $\mathrm{P}$ enriched areas are unchanged. An efflux of $\mathrm{Ca}^{2+}$ from microdomains not co-localized with $\mathrm{P}$ may contribute to the structural and functional deficits observed in nerve vulnerable to spreading damage following neurotrauma. NanoSIMS analyses of Ca microdomains allow quantitative and novel insights into Ca dynamics, applicable to a range of normal, as well as diseased or injured mammalian 20 systems.

\section{Introduction}

Rapid alterations in $\mathrm{Ca}^{2+}$ concentration and location are key features of the injury response in a range of biological systems. Injury to the central nervous system (CNS) results in damage to 25 neurons and glia, with rapid alterations in $\mathrm{Ca}^{2+}$ concentration thought to be an important contributor ${ }^{1-4}$. Undamaged tissue adjacent to the primary injury is also susceptible to cell death through a series of disruptive cellular and metabolic cascades, termed secondary degeneration ${ }^{5}$. Secondary degeneration is 30 thought to be triggered by glutamate-induced excitotoxicity, resulting in excess influx of $\mathrm{Ca}^{2+}$ into cells and associated oxidative stress ${ }^{6-8}$. While fluctuations in $\mathrm{Ca}^{2+}$ are a normal and essential part of neural function, excess $\mathrm{Ca}^{2+}$ influx into cells is a feature of CNS injury and is thought to contribute to the death of 35 neurons and glia vulnerable to secondary degeneration ${ }^{1,4}$. The dynamic changes in $\mathrm{Ca}^{2+}$ flux in tissue vulnerable to secondary degeneration have not been fully characterized in vivo. Nevertheless, it has been proposed that movement of $\mathrm{Ca}^{2+}$ between glia and neurons could play a part in the spread of $\mathrm{Ca}^{2+}$ ${ }_{40}$ from the primary injury site, to areas affected by secondary degeneration $^{9,10}$.

$\mathrm{Ca}^{2+}$ has been observed throughout the cytosol ${ }^{2}$, and in in vitro studies has also been shown to be localized in areas of increased concentration, referred to as microdomains ${ }^{11}$. ${ }_{45}$ Microdomains of $\mathrm{Ca}^{2+}$ are associated with presynaptic $\mathrm{Ca}^{2+}$ channels, as well as other cell features in the soma, including the mitochondria, Golgi body and nucleus ${ }^{11-13} \cdot \mathrm{Ca}^{2+}$ microdomains are involved in synaptic activation, AMP signaling and presynaptic transmitter release ${ }^{14}$ and are found in astrocyte 50 somata and processes ${ }^{13,15}$. In vivo and ex vivo 2-photon imaging has been used to demonstrate $\mathrm{Ca}^{2+}$ signaling using indicator dyes and genetically encoded $\mathrm{Ca}^{2+}$ indicators in normal and injured white matter tract ${ }^{16,17}$, including in astrocytes ${ }^{13,18-20}$, with the advantage of real-time recording ${ }^{21}$. However, the spatial 55 resolution of the resultant images is limited. Quantification of changes in $\mathrm{Ca}^{2+}$ signal associated with microdomains in individual cellular compartments has been confined to small transients in sub-regions of $4-12 \mu \mathrm{m}$ referred to as 'focal' events 19 , and $\mathrm{Ca}$ signals specifically associated with individual 60 microdomains are yet to be comprehensively characterized ${ }^{22}$. Furthermore, these techniques rely on indirect measures of $\mathrm{Ca}^{2+}$, detecting changes in the indicator, rather than changes in $\mathrm{Ca}^{2+}$ itself.

Here, we combine nanoscale secondary ion mass spectrometry ${ }_{65}$ (NanoSIMS) with immunohistochemistry to characterize Ca microdomains in normal optic nerve, quantitatively investigating changes in Ca microdomains specific to axons or glia vulnerable to secondary degeneration, in the 3 months after partial injury. NanoSIMS is a high resolution imaging mass spectrometry 70 technique that uses a finely focused ion beam to extract the top few atomic layers of a sample surface, producing an image reflecting the elemental/isotopic composition of the sample. It is 
highly versatile, and has been used to investigate cell structure ${ }^{23}$, and map the distribution of stable isotopes ${ }^{24,25}$, metal species ${ }^{26}$ and organometallic complexes ${ }^{27}$ within biological tissue. Furthermore, NanoSIMS has the important advantage of directly 5 measuring the abundance of ions such as $\mathrm{Ca}$, rather than relying on indirect measures of changes in indicator dyes. In a preliminary study, we used NanoSIMS to directly visualize the redistribution of areas of elevated $\mathrm{Ca}$ in optic nerve vulnerable to secondary degeneration within the first day of partial optic nerve 10 transection injury ${ }^{28}$.

In the current study we demonstrate that this high-resolution technique can also be used to quantify changes in Ca signals associated with individual microdomains as small as $1 \mu \mathrm{m}$ in diameter, specific to glial or neuronal regions identified 15 immunohistochemically. We define $\mathrm{Ca}$ microdomains as localised areas of increased $\mathrm{Ca}$ concentration likely to be associated with a $\mathrm{Ca}^{2+}$ signal, which can merge together to form larger microdomains or macrodomains ${ }^{14}$. The Ca microdomains we describe in the current study are distinct from Ca 20 nanodomains, which are highly localized, transient phenomena, smaller than $1 \mu \mathrm{m}$ and associated with a single ion channel ${ }^{29}$. It is important to note that our cryopreservation protocol does not include fixation, and as such, it is possible that transient $\mathrm{Ca}$ microdomains were captured at the moment of high-pressure 25 freezing. We assess fields of view from central optic nerve not damaged by the initial partial injury and shown to be vulnerable to secondary degeneration following dorsal partial transection ${ }^{30}$, 31. We examine ex vivo central optic nerve regions by rapid excision and cryopreservation of tissue, thereby ensuring that, as 30 far as possible, cellular activity is immobilized such that the tissue closely approximates its living state. A further novel advantage of our current application of NanoSIMS is that $\mathrm{Ca}$ microdomains can be categorized and quantified on the basis of their co-localization with nano-scale areas enriched in ${ }_{35}$ Phosphorus (P) and therefore with areas that are or are not likely to have a higher concentration of phosphorylated proteins. Receptor mediated events involving Ca release can be either dependent or independent of phosphorylation ${ }^{19,32,33}$. Changes in $\mathrm{Ca}^{2+}$ microdomains differentiated according to co-localization 40 with areas enriched in $\mathrm{P}$, may thus implicate changes in functionally important regulatory mechanisms in specific cell types vulnerable to secondary degeneration.

\section{Experimental}

\section{Animals and Partial Optic Nerve Transection}

45 Adult, female piebald viral Glaxo (PVG) black hooded rats (165205 g, Animal Resource Centre, Murdoch, WA, Australia) were used as described ${ }^{30}$. Procedures were approved by The University of Western Australia's Animal Ethics Committee and conformed to the National Health and Medical Research Council 50 of Australia guidelines on the use of animals in research. Partial optic nerve transection of the right optic nerve was performed as described previously ${ }^{30}$.

\section{Sample Preparation for NanoSIMS}

Optic nerve tissue segments were collected 5 min, 1, 3, 7 days, 1 55 and 3 month post partial optic nerve transection $(n=3-4 /$ time point). Segments $(200 \mu \mathrm{m})$ of right optic nerve from uninjured animals were used as controls $(n=3)$. Transverse segments of the right optic nerve encompassing the injury site $(200 \mu \mathrm{m})$ were rapidly excised using a custom-built cutter incorporating parallel 60 razor blades placed $200 \mu \mathrm{m}$ apart. The process of excision, cryoprotection and freezing was completed within 90 seconds. NanoSIMS analyses are similar to ultrastructural studies in that, due to practical considerations, only a relatively small number of samples can be assessed. Numbers of animals used per group for 65 this new application of NanoSIMS technology was based on previously published ultrastructural analyses ${ }^{36}$; blinding of investigators to sample identity was not possible. Subsequent to excision, each segment was briefly dipped in cryoprotectant solution (2\% low-temperature gelling agarose, Sigma, St. Louis, $70 \mathrm{MO})$, transferred to a membrane carrier ( $1.5 \times 0.2 \mathrm{~mm}$ diameter, Leica) and high-pressure frozen (EMPACT 2, Leica) using liquid $\mathrm{N}_{2}$, enabling a freezing rate of approximately $25,000{ }^{\circ} \mathrm{C} / \mathrm{sec}$ with minimal ice crystal formation. A protocol outlined by Clode and Marshall ${ }^{52}$ was used to minimize movement of mobile ions such 75 as Ca. In brief, frozen optic nerve samples were freeze-substituted (EM AFS2, Leica) in $10 \%$ acrolene in $\mathrm{Et}_{2} \mathrm{O}$ and tissue was infiltrated with Lowicryl HM20 resin (ProSciTech, Thuringowa, $\mathrm{QLD}$, Australia) in $\mathrm{Et}_{2} \mathrm{O}$ in line with the following procedure: freeze-substitution, $24 \mathrm{hr}$ at $-100^{\circ} \mathrm{C}, 168 \mathrm{hr}$ at $-90^{\circ} \mathrm{C}, 350 \mathrm{hr}$ at $8070^{\circ} \mathrm{C}$, and $24 \mathrm{hr}$ at $-50^{\circ} \mathrm{C}$; resin infiltration at $-50^{\circ} \mathrm{C}, 1 \mathrm{hr} 1: 1,1 \mathrm{hr}$ 1:2, 1 hr 100\% HM20, 24 hr 100\% HM20, followed by two $48 \mathrm{hr}$ $\mathrm{UV}$ polymerization steps at $-50{ }^{\circ} \mathrm{C}$ and $20^{\circ} \mathrm{C}$. The presence of water was minimized by preparing all solutions and performing freeze-substitution with molecular sieve (3A, Acros Organics). ${ }_{85}$ Optic nerve segments were dry serially sectioned with a diamond knife (Histo, Diatome): three $1 \mu \mathrm{m}$ sections were collected for toluidine blue staining for identification of the injury site; the next ten $1 \mu \mathrm{m}$ sections were collected in pairs - one for NanoSIMS and the next for immunohistochemical analyses; the 90 following $10 \mu \mathrm{m}$ were discarded. The pattern was repeated until the whole nerve segment had been serially sectioned. For each optic nerve segment, a single section at the injury site was selected for NanoSIMS analysis, transferred onto 1 inch diameter polished silicon wafers, and flattened/attached using a single drop 95 of dry tetrahydrofuran. The wafers were then coated with $10 \mathrm{~nm}$ $\mathrm{Au}$ to provide conductivity at high voltage in the NanoSIMS. Adjacent sections for immunohistochemical analyses were stored at room temperature.

\section{Measurement of samples using NanoSIMS}

100 For each optic nerve segment, sections were viewed to locate disturbance of the tissue indicating the dorsal injury site and a single section clearly at the injury site was chosen for analysis. One or more fields of view $(30 \mu \mathrm{m} \times 30 \mu \mathrm{m})$ in the central region of the selected section (and therefore in an area vulnerable to 105 secondary degeneration) were analyzed. Fields of view, including both axons and glia, were chosen using light-microscopy images of adjacent Toluidine blue-stained sections, in order to ensure a range of cellular features could be analyzed.

Elemental images were acquired using the CAMECA 110 NanoSIMS 50 ion microprobe at The University of Western Australia. Prior to imaging, all fields of view were implanted with a primary ion dose of $1 \times 10^{17}$ ions $/ \mathrm{cm}^{2}$, thereby affording a steady state of secondary ion yield. $\mathrm{A} \mathrm{Cs}^{+}$primary ion beam (nominal beam diameter $=100 \mathrm{~nm}$, current $=1.5 \mathrm{pA}$ ) was used to 115 sputter the negative ion species ${ }^{12} \mathrm{C}^{12} \mathrm{C},{ }^{12} \mathrm{C}^{14} \mathrm{~N},{ }^{31} \mathrm{P},{ }^{32} \mathrm{~S},{ }^{35} \mathrm{Cl}$ and 
secondary electrons. An $\mathrm{O}^{-}$primary ion beam (nominal beam diameter $=600 \mathrm{~nm}$, current $=28 \mathrm{pA}$ ) was then used to sputter the positive ion species ${ }^{12} \mathrm{C},{ }^{23} \mathrm{Na},{ }^{39} \mathrm{~K}$ and ${ }^{40} \mathrm{Ca}$. All fields of view were imaged at a resolution of $256 \times 256$ pixels, with a dwell 5 time of $30 \mathrm{~ms} /$ pixel. There were no significant isobaric interferences, so it was not necessary to tune the mass spectrometer to high mass resolution; charge build-up during $\mathrm{Cs}^{+}$ primary beam use was not observed. The combination of underlying Si substrate and overlying Au coat provided adequate

10 sample conductivity. As the valency of the detected ions is not discernible, detected ions are referred to by their isotopic species (e.g. ${ }^{40} \mathrm{Ca}$ ) and elemental symbol (Ca), not oxidation state (e.g. $\mathrm{Ca}^{2+}$.

\section{Analysis of NanoSIMS data}

15 The NanoSIMS produces secondary ion maps with 32-bit data density, where each pixel records the number of secondary ion counts within the acquisition time. Secondary ion images were corrected for detector deadtime (44 ns) and analyzed using the OpenMIMS plugin for Fiji/ImageJ (version 2.0; NIH). ${ }^{23}$ For 20 quantitative analysis of ${ }^{40} \mathrm{Ca}$, area (pixels) and mean secondary ion count (counts/pixel) data for specified regions-of-interest within the image were extracted. For analysis, area and mean counts were converted to $\mu \mathrm{m}^{2}$ (72.817 pixels $^{2} / \mu \mathrm{m}^{2}$ ).

In order to correct for any signal strength variability from 25 sample to sample, each ion-signal from which data were extracted and analyzed quantitatively was displayed as a ratio of the ${ }^{12} \mathrm{C}$ signal collected for the entire field of view.

To allow for easier visualization of structural information, overlays of three secondary ion images, to which each had been 30 assigned one RGB color channel, were constructed (Fig. 1A and B). Ten secondary ion channels combined into 3 channel RGB color images result in 720 unique permutations, shown are two possibilities. Changes in the displayed color were indicative of changes in the ratio of one element relative to others. Overlays of ${ }^{35}{ }^{31} \mathrm{P},{ }^{32} \mathrm{~S}$ and ${ }^{12} \mathrm{C}^{14} \mathrm{~N}$ secondary ion images (P-S-CN assigned to RGB color channels in order listed) were found to best visually enhance structural information (nuclei, axons, myelin) present in individual images.

Ca microdomains were defined as any area or group of pixels ${ }_{40}$ with a ${ }^{40} \mathrm{Ca}$ signal in excess of 20 secondary ion counts/pixel, with regions of punctate or 'speckled' $\mathrm{Ca}$ defined as $0 \leq 20$ secondary ion counts/pixel. Thresholds set to these values were used to generate regions-of-interest using Fiji/ImageJ, allowing data extraction only within, or outside, Ca microdomains.

${ }_{45}$ Microdomain/punctate regions-of-interest were exported as black/white bitmap images (Fig. 1C). Ca microdomains were further divided into those within axonal or glial regions: identified by reference to secondary electron images, P-S-CN overlays and immunohistochemically stained adjacent sections. 50 Axonal/glial regions were also exported as black/white bitmap images (Fig. 1D). Some Ca microdomains co-localized with areas of enriched $\mathrm{P}$ signal, observed as small pink dots in P-S-CN images. Therefore, where appropriate, $\mathrm{Ca}$ microdomains are hereafter referred to as non-P co-localized or P co-localized Ca

55 microdomains to reflect their location relative to these $\mathrm{P}$ enriched areas, and were treated as separate $\mathrm{Ca}$ microdomain types. Exported black/white bitmaps were modified in the GNU Image Manipulation Program (2.8.x), assigning different shades of grey (in hexadecimal) to the following region of interest types: glial 60 punctate (000000), glial P associated Ca microdomains (4c4c4c), glial non-P Ca microdomains (666666), axonal non-P associated Ca microdomains (сссссC), axonal $\mathrm{P}$ associated Ca microdomains (e6e6e6) and axonal punctate (ffffff) (Fig. 1E). The resulting bitmaps were used to define regions-of-interest in Fiji/ImageJ 65 indicative of tissue type, allowing data to be extracted separately for the different Ca distribution types (Fig. 1F). Data for Ca and $\mathrm{C}$ were extracted separately. Mean signal (counts/pixel) and area (pixels) were used to calculate total signal, from which ${ }^{40} \mathrm{Ca} /{ }^{12} \mathrm{C}$ ratio was determined.
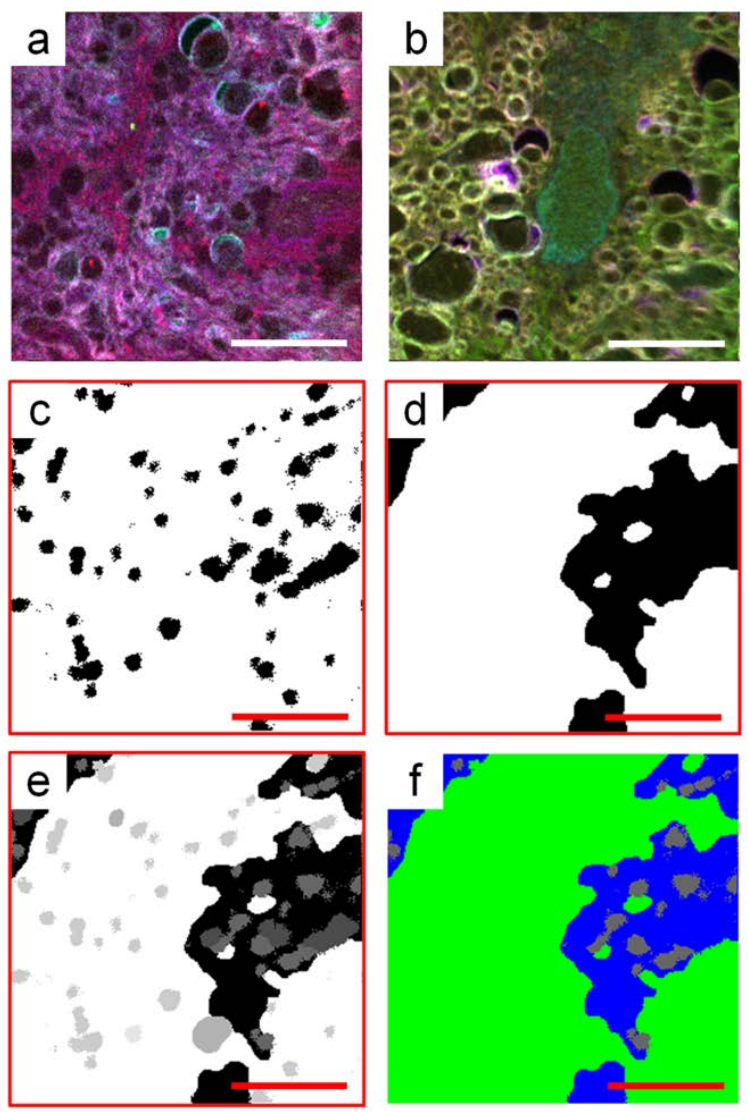

Fig1 Pictorial representation of image analysis and data extraction process. Selected permutations of combinations of 3 channel RGB color images: Cl-P-S (A) and S-Cl-P (B). Ca microdomain (C, in black) and tissue type $(\mathrm{D}$, glial = black; axonal $=$ white $)$ can be used to generate 8-bit greyscale maps (E) used to define individual regions of interest in

75 Fiji/Image-J: example shown (F; non-P co-localized glial microdomains, color code $=666666$; green and blue are excluded regions). All images 30 $\mu \mathrm{m} \times 30 \mu \mathrm{m}$, scale bar $=10 \mu \mathrm{m}$.

The number of Ca microdomains was counted manually and expressed as a density, relative to the area $\left(\mu \mathrm{m}^{2}\right)$ of axonal or glial 80 regions. Clustered Ca microdomains were counted as separate if there was a clearly defined area of lower signal between two points of higher signal. In order to compare the relative areas of non-P or P co-localized Ca microdomains, Ca microdomain areas were expressed as a proportion of the total area of the tissue type 85 (axonal or glial) in which they were found. The total area (of the pre-determined region-of-interest) and the sum of ${ }^{40} \mathrm{Ca}$ and of ${ }^{12} \mathrm{C}$ signal/pixel (counts/pixel within area of predetermined region-ofinterest) were determined using the OpenMIMS plugin. The mean \pm S.E.M. size of Ca microdomains were expressed in $\mu \mathrm{m}^{2}$ 
and were calculated by dividing the total area of $\mathrm{P}$ or non-P colocalized $\mathrm{Ca}$ microdomains by the number of these $\mathrm{Ca}$ microdomains. The relative $\mathrm{Ca}$ concentration of the $\mathrm{Ca}$ microdomains is expressed as mean ${ }^{40} \mathrm{Ca} /{ }^{12} \mathrm{C}$ ratio \pm S.E.M / unit 5 area $\left(\mu \mathrm{m}^{2}\right)$ of the tissue type in which the Ca microdomains were found.

Quantification of punctate Ca signal that was not part of Ca microdomains was conducted on whole images. Nuclei were identified with reference to Hoechst stained adjacent sections and 10 P-S-CN overlays, and were excluded from these analyses. The mean ${ }^{40} \mathrm{Ca} /{ }^{12} \mathrm{C}$ ratio \pm S.E.M was normalized for the punctate area $\left(\mu \mathrm{m}^{2}\right)$ in each image. Visualization of the punctate Ca signal was achieved by adjusting the displayed minimum-maximum grayscale values to correspond to 0 - 60 in 32-bit ${ }^{40} \mathrm{Ca} /{ }^{12} \mathrm{C}$ ratio 15 images (scale factor of 10) generated using the OpenMIMS plugin.

No marked differences in mean densities, size or intensities of Ca microdomains were observed between multiple fields of view within a single optic nerve section, although the nature of the 20 single observations generated per region-of-interest precluded statistical confirmation of their similarity. Therefore, mean values for all outcomes were generated for the 1 - 3 fields of view analysed/optic nerve (therefore generating one value/animal for each outcome measure, $\mathrm{n}=$ number of animals) and grand means 25 and standard errors of the mean (S.E.M.) for all animals/time point were calculated. Of necessity, a microanalysis technique such as NanoSIMS must assess a small fraction of the available tissue section. As the frequency of Ca microdomains is relatively low, there is some variability between replicates. Variation could 30 only have been reduced by substantially increasing the number of fields of view sampled, resulting in a reduction in the number of time points post injury that could be assessed, due to the practical space, time and cost limitations of the analysis technique. The decision was made to maintain the chosen study design and 35 appropriate statistical analyses has enabled interpretation of the data. Due to the relatively small sample size, data was assumed to be normally distributed. Values were statistically analyzed using 2-way ANOVA, ANOVA and Bonferroni/Dunn post-hoc tests (IBM SPSS Statistics Version 20; IBM Corporation, Armonk, $40 \mathrm{NY}$ ) as applicable, requiring a significance of $\mathrm{P} \leq 0.05$. Lavene's test of equal variance was used to show that population variances at different time points were not significantly different $(\mathrm{P}>0.05)$. Immunohistochemistry

Sections adjacent to NanoSIMS sections were analyzed 45 immunohistochemically according to previously described procedures ${ }^{30}$, with the following modifications. Sections were etched in saturated $\mathrm{NaOH} / 100 \% \mathrm{EtOH}$ for 2 minutes and graded through an $\mathrm{EtOH}$ series followed by microwave antigen retrieval in $10 \mathrm{mM}$ citric acid buffer, $\mathrm{pH}$ 6.0. Primary antibodies (diluted 50 in PBS $+0.2 \%$ Triton X100 (PBST) containing 5\% normal donkey serum) were used to detect: neurons (anti- $\beta$-III tubulin; 1:500, Tuj1 clone Covance or ab18207 Abcam); myelin basic protein (anti-MBP; 1:500, ab40390 Abcam); astrocytes (antiGFAP; 1:500, GA5 Millipore); $\mathrm{Ca}^{2+} /$ calmodulin-dependent 55 protein kinase II (anti-CaMKII; 1:100, ab2724 Abcam) or voltage-dependent anion channel 2 (anti-VDAC2; 1:200, HPA043475 Sigma). Antibody binding was visualized following $2 \mathrm{~h}$ incubation at room temperature with appropriate secondary antibodies (Alexa Fluor 488, 555 or 647 (Molecular Probes) all at 60 1:400 in PBST). Nuclei were identified using Hoechst stain (10 $\mu \mathrm{g} / \mathrm{mL}$, Invitrogen). Slides were coverslipped using Fluoromount$\mathrm{G}$ (Southern Biotechnology) and visualized and imaged using a Nikon Eclipse Ti inverted microscope (Nikon Corporation, Japan) with a 40X/1.3 N.A. oil immersion objective or a Leica ${ }_{65}$ TCS SP2 multiphoton confocal microscope (Leica Microsystems Heidelberg GmbH, Germany) with a PL APO CS 63.0X/1.40 oil immersion objective. All images collected using Nikon Elements AT software were deconvoluted using autoquant blind deconvolution. All sections for each antibody were 70 immunostained at the same time to ensure uniformity of the immunohistochemical procedures, and images were captured at constant exposure times. Control sections stained only with secondary antibodies were included in all experiments and showed no or only minimal fluorescence (data not shown).

\section{${ }_{75}$ Results}

NanoSIMS characterization of Ca microdomains in normal optic nerve

In normal optic nerve excised from uninjured animals, NanoSIMS secondary ion imaging reveals the distribution of $\mathrm{Ca}$ 80 either as localized, high intensity circular features as small as 1 $\mu \mathrm{m}$ in diameter, henceforth referred to as $\mathrm{Ca}$ microdomains (Fig. 2A) ${ }^{11}$, or as a disseminated, low intensity, background signal with a speckled appearance, referred to as punctate.

A signal threshold was used to delineate Ca microdomains for
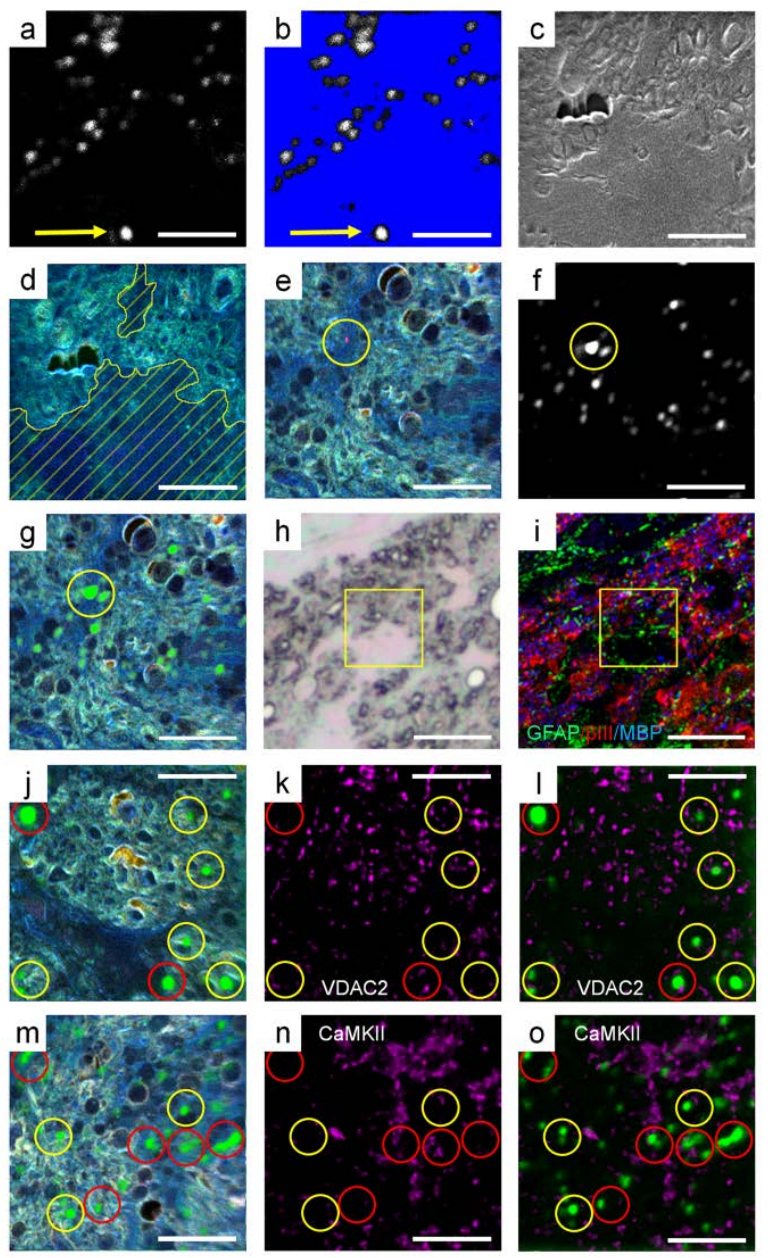
Fig.2 NanoSIMS image of normal ON illustrating ${ }^{40} \mathrm{Ca} /{ }^{12} \mathrm{C}$ ratio (A), with Ca microdomain threshold set (B, area in blue is the excluded region). Yellow arrows indicate a representative Ca microdomain (A, B).

Structural information can be visualized in images generated from 5 secondary electrons and from RGB overlays of ${ }^{31} \mathrm{P},{ }^{32} \mathrm{~S}$ and ${ }^{12} \mathrm{C}^{14} \mathrm{~N}$ (P-S$\mathrm{CN}$ ) signal in the same field of view (C, D). P-S-CN images also allow for identification of areas enriched in $\mathrm{P}$ (E, pink dot), which in some cases co-localize with Ca microdomains ( $\mathrm{F}$, overlaid in $\mathrm{G}$ ), circled in yellow. Light microscopy $(\mathrm{H})$ along with immunohistochemical staining (I) of 10 sections encompassing the NanoSIMS field of view (boxed in yellow) allow visualization of structural features that were identified as glial regions containing GFAP+ astrocytes (green) or axonal regions containing $\beta$-III tubulin+ (red) axonal profiles associated with MBP+ myelin (blue) (I). Glial (hashed in yellow) and axonal regions can then be 15 identified in P-S-CN images (D). Images of Ca microdomains overlaid on P-S-CN images (J) together with immunohistochemical staining for VDAC2 (K), demonstrate co-localization of some Ca microdomains (green) with VDAC2 (purple) (L). Both non-P co-localized (circled in yellow) and $\mathrm{P}$ co-localized (circled in red) Ca microdomains were found 20 to associate with VDAC2. Images of Ca microdomains overlaid on P-S$\mathrm{CN}$ images (M) together with immunohistochemical staining for CaMKII

(N) show that Ca microdomains (green, non-P co-localized circled in yellow, P co-localized circled in red) do not appear to co-localize with CaMKII (purple) $(\mathrm{O})$. Images $\mathrm{H}$ and I, scale bar $=30 \mu \mathrm{m}$. All other NanoSIMS images $30 \mu \mathrm{m} \times 30 \mu \mathrm{m}$, scale bar $=10 \mu \mathrm{m}$.

quantification purposes (Fig. 2B). Secondary electron NanoSIMS images (Fig. 2C) allowed visualization of morphological regions that corresponded to specific colored regions in RGB overlays of ${ }^{31} \mathrm{P},{ }^{32} \mathrm{~S}$ and ${ }^{12} \mathrm{C}^{14} \mathrm{~N}$ (P-S-CN) images (Fig. 2D), indicating 30 differences in elemental composition. Some Ca microdomains were co-localized with areas of enriched ${ }^{31} \mathrm{P}$ signal, observed as small pink dots in P-S-CN images (Fig. 2E, co-localization illustrated in Fig. 2F, G, larger microdomain of those circled in yellow), whereas others did not (Fig. 2E - G, smaller 35 microdomain of those circled in yellow). Ca microdomains that were or were not co-localized with P-enriched areas (referred to henceforth as $\mathrm{P}$ or non-P co-localized) were quantified separately for axonal and glial regions (see below). Distribution of calcium in the various forms of Ca microdomains is illustrated (Fig. 3).

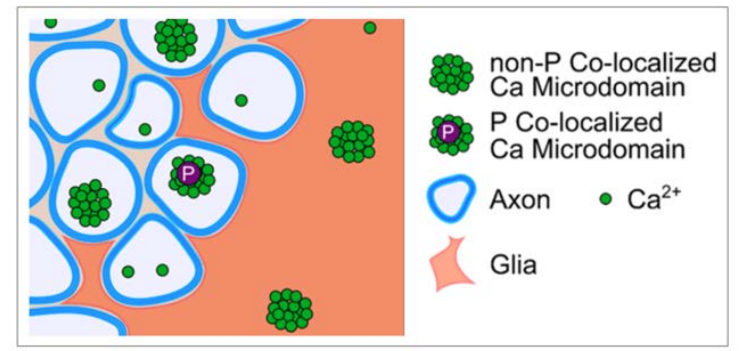

$40 \quad$ Fig.3 Schematic illustrating the distribution of $\mathrm{Ca}$ in $\mathrm{P}-$ and non-P colocalized microdomains in axonal and glial regions.

We first investigated the sub-cellular localization of $\mathrm{P}$ and nonP Ca microdomains within the normal optic nerve. $\mathrm{Ca}^{2+}$ microdomains have been proposed to be associated with the 45 plasma membrane ${ }^{11}$ and intracellular structures including endoplasmic reticulum and mitochondria ${ }^{13}$. To confirm this using our technique and to additionally determine whether $\mathrm{P}$ and non-P co-localized Ca microdomains are located in different subcellular regions, P-S-CN images overlaid with ${ }^{40} \mathrm{Ca} /{ }^{12} \mathrm{C}$ images 50 (Fig. 2J, M) were compared to adjacent sections immunohistochemically identifying VDAC2 (labels the mitochondrial membrane; Fig. 2K, L) or CaMKII (located at the plasma membrane when activated, Fig. 2N, O). As a kinase, we hypothesized that CaMKII may be selectively associated with $\mathrm{P}$ 55 co-localized Ca microdomains. Immunohistochemical analyses could not be conducted on the same sections imaged using NanoSIMS due to the required $\mathrm{Au}$ coating of the sample. However, comparison of adjacent sections allows an indication of possible co-localization of VDAC2 and/ or CaMKII with the Ca 60 microdomains. The majority of Ca microdomains in normal optic nerve were closely associated but not precisely co-localized with VDAC2 (circled in yellow for non-P and in red for P co-localized areas), consistent with Ca accumulation in mitochondria ${ }^{34}$; there was no indication of selective association for non-P or $\mathrm{P}$ co${ }_{65}$ localized Ca microdomains. Quantitative analysis of colocalization between NanoSIMS Ca microdomains and VDAC2 staining would require confocal imaging and analysis of a single image in the z plane. As such, it is not possible to conduct colocalization analyses in the current study as 70 immunohistochemical and NanoSIMS analyses can only be conducted on adjacent sections. Furthermore, we did not observe precise co-localization, so co-localization values are likely to be negligible. There appeared to be no consistent association of either non-P co-localized (circled yellow) or P co-localized 75 (circled red) Ca microdomains with CaMKII.

Light microscopy images and immunohistochemical analyses of sections adjacent to those used for NanoSIMS analysis also allowed for differentiation of glial and neuronal cell types (Fig. $2 \mathrm{H}, \mathrm{I})$. The relatively featureless areas in the secondary electron 80 images and darker areas in the P-S-CN images corresponded to areas containing GFAP+ astrocytes, a subset of glial cells (Fig. 2D, hashed in yellow, determined with reference to Fig. $2 \mathrm{H}$ and I), whereas circular features corresponded to $\beta$-III tubulin+ axons closely associated with MBP+ myelin (Fig. 2D, I). Quantification 85 and measurement of non-P and P co-localized Ca microdomains was therefore conducted separately in axonal and glial regions, based on comparisons with P-S-CN images of the same field of view (Fig. 4).

To compare the proportion of non-P and P co-localized Ca 90 microdomains within each tissue type (glial and axonal), the area of Ca microdomains was expressed as a proportion of the total area of the tissue type in which the Ca microdomains were found (glial or axonal). In normal optic nerve, glial regions contained a significantly greater proportion of non-P co-localized $\mathrm{Ca}$ 95 microdomains than P-localized Ca microdomains (Fig. 4A, P $\leq$ 0.05 ), while there was no significant difference between the microdomains in axonal regions (Fig. 4B, P > 0.05). Comparison of the proportion of Ca microdomains between the tissue types revealed that there was a significantly greater proportion of non-P 100 co-localized $\mathrm{Ca}$ microdomains in glial regions than axonal regions (Fig. 5A, $\mathrm{P} \leq 0.05$ ).

\section{Density of a subtype of Ca microdomains was reduced during secondary degeneration}

To directly quantify changes in Ca microdomains vulnerable to 105 spreading damage following neurotrauma, we assessed the density of Ca microdomains in glial and axonal regions from central optic nerve, undergoing secondary degeneration following dorsal partial nerve injury (Fig. 4C-H) ${ }^{30,31}$. In glial regions, the total density of Ca microdomains (both non-P and P co-localized) 110 significantly decreased at 5 minutes, 1, 3, 7 days and 1 month 

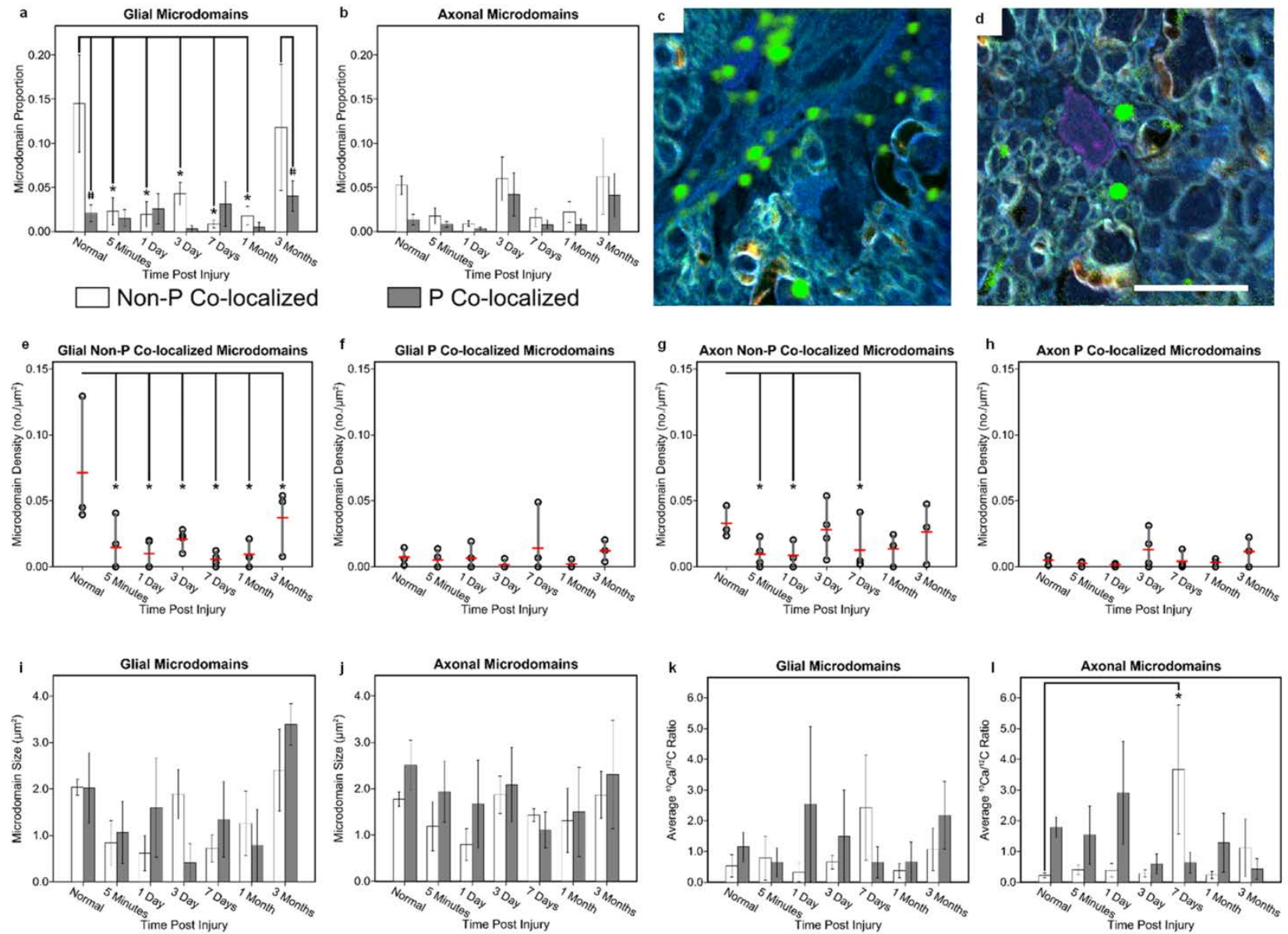

Fig.4 Histograms show mean Ca microdomain proportions \pm S.E.M. in glial (A) and axonal (B) regions. Representative NanoSIMS images illustrate the changes in various microdomain types observed in normal optic nerve (C) and at 1 day following partial optic nerve transection (D). Scale bar $=10 \mu \mathrm{m}$. Dot plots show quantification of mean non-P (E) and P (F) co-localized Ca microdomain density in glial regions, and non-P (G) and P (H) co-localized Ca microdomain density in axonal regions (number $\left./ \mu \mathrm{m}^{2}\right)$. Significant differences compared with normal optic nerve are indicated $(* \mathrm{P} \leq 0.05)$. Black circles 5 indicate mean density for each animal at a particular time point, grey vertical bars illustrate the range and red horizontal bars indicate grand means for each time point. Histograms show: size of non-P and P co-localized Ca microdomains in glial (I) and axonal (J) regions (in $\mu \mathrm{m}^{2}$ ). The mean ${ }^{40} \mathrm{Ca} /{ }^{12} \mathrm{C}$ ratio \pm S.E.M. of non-P and P co-localized Ca microdomains in glial $(\mathrm{K})$ and axonal (L) regions (in arbitrary units). Significant differences between values at each time-point are indicated by \# $(\mathrm{P} \leq 0.05)$ and significant changes as a consequence of injury are indicated by * $(\mathrm{P} \leq 0.05)$.

after injury, compared to normal optic nerves (control from 10 uninjured normal animals) (normal $0.058 \pm 0.017 ; 5$ minutes $0.016 \pm 0.006 ; 1$ day $0.013 \pm 0.005 ; 3$ day $0.032 \pm 0.009 ; 7$ day $0.018 \pm 0.009 ; 1$ month $0.014 \pm 0.005, \mathrm{P} \leq 0.05: 3$ month $0.044 \pm$ $0.012, \mathrm{P}>0.05)$. When the densities of non-P co-localized $\mathrm{Ca}$ microdomains were analysed separately, significant decreases 15 were observed at 5 minutes, 1, 3, 7 days, 1 and 3 months after injury, compared to normal optic nerves (Fig. $4 \mathrm{E}, \mathrm{P} \leq 0.05$ ). No significant differences in glial $\mathrm{P}$ co-localized $\mathrm{Ca}$ microdomains were observed at any of the time points assessed after injury (Fig. $4 \mathrm{~F}, \mathrm{P}>0.05)$. In axonal regions, a similar trend was observed: the 20 density of non-P co-localized $\mathrm{Ca}$ microdomains significantly decreased at 5 minutes, 1 and 7 days post injury (Fig. 4G, $\mathrm{P} \leq$ 0.05 ) and there were no significant changes in the density of $\mathrm{P}$ co-localized axonal microdomains compared to normal (uninjured control) optic nerves (Fig. 4H, P > 0.05). Total Ca 25 microdomain density in axonal regions (sum of non-P and $\mathrm{P}$ colocalized) did not change following injury (data not shown, $\mathrm{P}>$ 0.05); indicating that pooling the $\mathrm{P}$ and non-P co-localized $\mathrm{Ca}$ microdomains together masked the significant changes observed in the non-P co-localized microdomains in axonal regions. The 30 described changes in Ca microdomain density can be observed in representative images in Figure 4C-D, with noticeably fewer microdomains at 1 day after injury (Fig. 4D) compared to normal optic nerve (Fig. 4C).

Changes in the proportion of non-P and $\mathrm{P}$ co-localized $\mathrm{Ca}$ 35 microdomains following partial optic nerve transection were consistent with the changes in density described above. Post injury, the glial regions exhibited a significant decrease in proportion of non-P Ca microdomains at all time points up to 3 months (Fig. 4A, $\mathrm{P}>0.05$ ), while the $\mathrm{P}$ co-localized 40 microdomains remained at normal levels at all time points after injury. As such, the proportion of non-P co-localized Ca microdomains was no longer greater than the proportion of $\mathrm{P}$ colocalized microdomains until 3 months after injury. Note that the apparently greater proportion of non-P than $\mathrm{P}$ co-localized $\mathrm{Ca}$ 45 microdomains in glial regions at day 3 (Fig. 4A) did not reach statistical significance due to the variability inherent in the data 
set as a whole. The proportion of axonal non-P or P co-localized Ca microdomains did not change significantly, relative to normal optic nerve, at any time point post injury (Fig. 4B, P > 0.05).

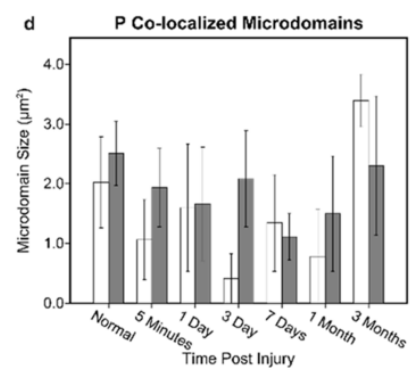

$\square$ Axonal Microdomains

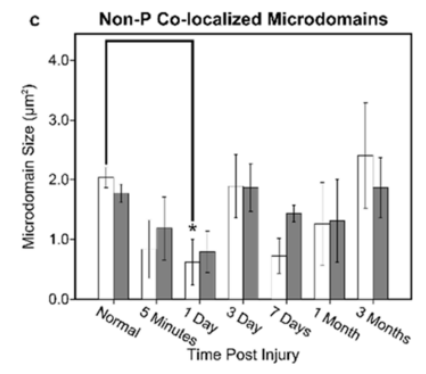

$\square$ Glial Microdomains

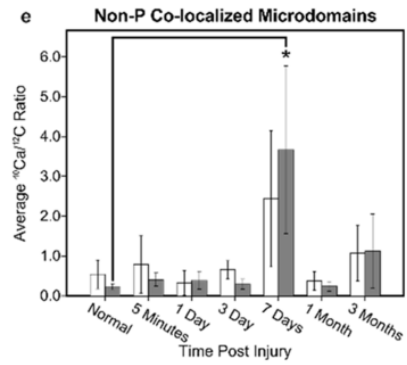

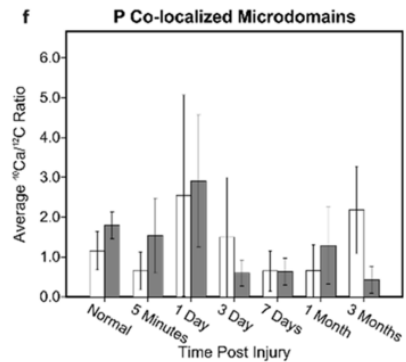

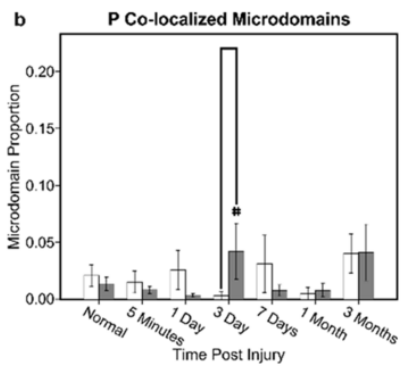

Fig.5 Histograms show: mean Ca microdomain proportions \pm S.E.M. in non-P (A) and P (B) co-localized Ca microdomains; size of glial and axonal non-P

(C) and P co-localized (D) Ca microdomains (in $\mu \mathrm{m}^{2}$ ). The mean ${ }^{40} \mathrm{Ca} /{ }^{12} \mathrm{C}$ ratio \pm S.E.M. of glial and axonal non-P (E) and P co-localized (F) Ca microdomains (in arbitrary units). Significant differences between values at each time-point are indicated by \# $(\mathrm{P} \leq 0.05)$ and significant changes as a consequence of injury are indicated by $*(\mathrm{P} \leq 0.05)$.

10 As a consequence of the reduction in the proportion of non-P colocalized $\mathrm{Ca}$ microdomains in glia after injury, there was no longer a significant difference in the proportions of these microdomains between axons and glia (Fig. 5A, $\mathrm{P}>0.05$ ). The only significant difference between glial and axonal regions in 15 terms of the proportion of P co-localized Ca microdomains, was a slightly greater proportion in axonal Ca microdomains at day 3 (Fig. 5B, $\mathrm{P} \leq 0.05$ ). There were no apparent differences in the association of $\mathrm{P}$ or non-P lo-localized $\mathrm{Ca}$ microdomains with VDAC2 or CaMKII after injury (Fig. 6, day 7 shown compared 20 to Fig. 2J-O).
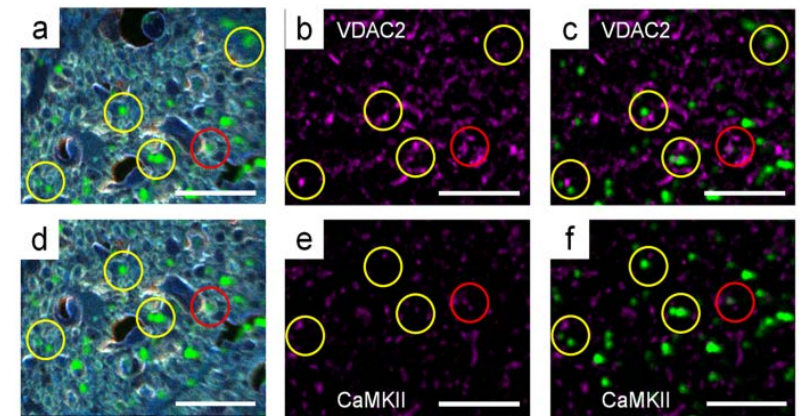

Fig.6 Images of Ca microdomains overlaid on P-S-CN images (A) together with immunohistochemical staining for VDAC2 (B), indicate colocalization of some Ca microdomains (green) with VDAC2 (purple) (C).

25 Both non-P co-localized (circled in yellow) and P co-localized (circled in red) Ca microdomains were found to associate with VDAC2. Images of

Ca microdomains overlaid on P-S-CN images (D) together with immunohistochemical staining for CaMKII (E) show that Ca microdomains (green, non-P co-localized circled in yellow, P co-localized 30 circled in red) do not appear to co-localize with CaMKII (purple) (F), scale bar $=10 \mu \mathrm{m}$.
Ca microdomain size and signal intensity in optic nerve vulnerable to secondary degeneration

Assessment of the size of non-P and $\mathrm{P}$ co-localized $\mathrm{Ca}$ 35 microdomains assessed individually for glial and axonal compartments (Fig. 4I, J) showed that there were no significant changes in the mean size of $\mathrm{Ca}$ microdomains as a result of partial optic nerve transection (Fig. 4I, J, P > 0.05), illustrated in representative images at 1 day after injury (Fig. 4D) compared to 40 normal optic nerve (Fig. 4C). There were also no significant differences between the size of non-P compared to P co-localized Ca microdomains in normal optic nerve or at any time after injury (Fig. 4I, J, P > 0.05), or between the size of Ca microdomains in axonal compared to glial regions (Fig. 5C, D, P > 0.05). Note that 45 the size of non-P co-localized Ca microdomains in glial regions at day 1 after injury was reduced when ANOVA was confined to non-P co-localized microdomains (Fig. $5 \mathrm{C}, \mathrm{P} \leq 0.05$ ). It is worth noting that the $\mathrm{O}$ beam used to detect $\mathrm{Ca}$ in the NanoSIMS is larger than the Cs beam used to detect the P-S-CN structural 50 information. Therefore, the Ca microdomains may appear larger in our NanoSIMS images than they actually are.

The mean ${ }^{40} \mathrm{Ca} /{ }^{12} \mathrm{C}$ ratio was assessed as an indication of the amount of $\mathrm{Ca}$ in the microdomains. A significant increase was observed in the $\mathrm{Ca}$ in axonal non-P co-localized $\mathrm{Ca}$ 55 microdomains at 7 days post injury, relative to normal optic nerve (Fig. 4L, $\mathrm{P} \leq 0.05$ ); no differences were observed in glial non-P co-localized Ca with injury (Fig. 4K, $\mathrm{P}>0.05$ ). No significant changes in Ca were observed for glial or axonal $\mathrm{P}$ co-localized $\mathrm{Ca}$ microdomains following injury (Fig. $4 \mathrm{~K}, \mathrm{~L}, \mathrm{P}>0.05$ ). Ca in $\mathrm{P}$ co60 localized $\mathrm{Ca}$ microdomains was not different to that in non-P colocalized in normal optic nerve or at any time after injury for 
either axonal or glial regions (Fig. 4K, L, P > 0.05). Similarly, Ca in axonal regions was not different to that in glial regions for either non-P or P co-localized Ca microdomains (Fig. 5E, F, P > 0.05). The mean punctate ${ }^{40} \mathrm{Ca} /{ }^{12} \mathrm{C}$ ratio was quantified in normal 5 optic nerve and at each time point post injury, but no significant differences were observed ( $\mathrm{P}>0.05$, data not shown).

\section{Discussion}

$\mathrm{Ca}^{2+}$ changes as a consequence of injury to the CNS are complex 1, rapid $^{3}$ and result in cellular degeneration and death ${ }^{1,2,4,35}$, but 10 have generally been viewed at a whole cell rather than a $\mathrm{Ca}^{2+}$ microdomain level. Using NanoSIMS combined with immunohistochemical identification of individual cell types in $e x$ vivo tissue samples, we have identified novel sub-populations of Ca microdomains that are or are not co-localized with areas 15 enriched in $\mathrm{P}$. We show for the first time that non-P and P colocalized Ca microdomains are differentially affected by injury. NanoSIMS technology has thus provided new insights into the chemistry of Ca microdomains, of broad relevance to mammalian systems.

20 The absence of significant changes in $\mathrm{P}$ co-localized $\mathrm{Ca}$ microdomains suggests that these $\mathrm{Ca}$ microdomains are not affected by secondary injury mechanisms, at least at the level we can currently detect. Indeed, secondary degeneration resulted in selective changes to non-P co-localized Ca microdomains. While 25 there is no doubt that protein phosphorylation plays a key role in regulation of diverse cellular processes, non-catalytic (non-P associated) functions of $\mathrm{Ca}^{2+}$ associated protein kinases such as phosphatidylinositol 3-kinase are increasingly recognized as important $^{32}$ and may be selectively disrupted when associated 30 with Ca microdomains in glia undergoing secondary degeneration. It is conceivable that $\mathrm{Ca}^{2+}$ is released from these non-P co-localized microdomains into the intra- and/or extracellular space, early after injury (modeled in Fig. 7), although current methodology does not permit quantification of 35 this phenomenon (discussed further below). Altered intracellular $\mathrm{Ca}^{2+}$ concentrations have been linked to oxidative stress and may contribute the structural and functional deficits of secondary degeneration $^{1,4,30,36}$.
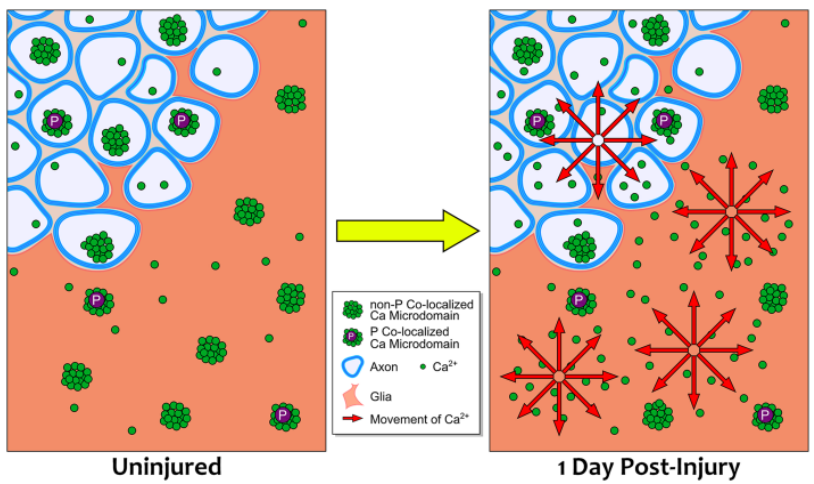

$40 \quad$ Fig.7 Model illustrating potential consequences of Ca microdomain changes in axonal and glial regions early post injury (1 day), compared to normal optic nerve. There is a decrease in the number of both axonal and glial non-P co-localized microdomains with injury, which may result in increased release of free (punctate) Ca into the cytosol and / or

45 extracellular space.
The combination of NanoSIMS with immunohistochemistry or cell labeling techniques has recently been reported ${ }^{37,38}$, but to our knowledge, ours is the first study combining direct high resolution NanoSIMS visualization of $\mathrm{Ca}$ microdomains with 50 immunohistochemical identification of individual cell types in $e x$ vivo mammalian tissue samples. This new technique allows for the measurement and quantification of $\mathrm{Ca}^{2+}$ microdomains in specific cell types in tissue and allowed demonstration that the changes in Ca microdomains were more pronounced in glial than 55 axonal compartments, perhaps reflecting the reactivity of astrocytes following injury to the CNS ${ }^{39}$ and propagation of $\mathrm{Ca}^{2+}$ waves through the astrocytic syncytium ${ }^{9,10} \cdot \mathrm{Ca}^{2+}$ microdomains can be transient phenomena ${ }^{40}$, however the rapid cryopreservation of tissue for NanoSIMS analysis allows their 60 accurate visualization at a particular point in time. Nevertheless, a limitation of our technique is that real-time assessments of $\mathrm{Ca}^{2+}$ signaling dynamics cannot be conducted. We also cannot guarantee that the sample preservation has not affected the chemistry of the samples, but we are confident in the results 65 given our rapid cryopreservation technique, combined with lowtemperature substitution and embedding ${ }^{41}$.

In vitro investigations designed to model the injury scenario have revealed that release of excess, localized $\mathrm{Ca}^{2+}$ from $\mathrm{Ca}^{2+}$ microdomains ${ }^{42}$, together with $\mathrm{Ca}^{2+}$ influx from the extracellular 70 space, leads to increased intracellular $\mathrm{Ca}^{2+}$ concentrations, oxidative stress and death of neurons and glia ${ }^{43-45}$. The role played by $\mathrm{Ca}^{2+}$ microdomains in the context of secondary injury in the CNS has not been studied extensively, perhaps due to the difficulty in studying $\mathrm{Ca}^{2+}$ microdomains in situ as opposed to 75 global $\mathrm{Ca}^{2+}$ levels ${ }^{22,46}$. The decrease in Ca microdomain density we have observed in axonal and glial regions vulnerable to secondary degeneration would suggest a decrease in overall $\mathrm{Ca}^{2+}$ sequestered in microdomains, with subtle differences between cell types.

80 Given the overall increase in $\mathrm{Ca}^{2+}$ concentration that has been reported in injured spinal cord ${ }^{47,48}$, particularly at the impact site 49 , our data suggest an efflux of $\mathrm{Ca}^{2+}$ from microdomains into a global punctate distribution, similar to that which has been observed from $\mathrm{Ca}^{2+}$ microdomains associated with mitochondria, 85 sarcoplasmic and endoplasmic reticulum in cultured HeLa cells and myocytes ${ }^{42,}{ }^{46}$. Released $\mathrm{Ca}^{2+}$ could exist intra- or extracellularly and the current NanoSIMS analyses do not distinguish between these; increasing the lateral resolution may increase sensitivity for this outcome in future assessments. The 90 NanoSIMS acquisition parameters for the current study were designed to allow quantification of Ca microdomains rather than the punctate ${ }^{40} \mathrm{Ca} /{ }^{12} \mathrm{C}$ signal; adjustments to sensitivity may reveal alterations in punctate ${ }^{40} \mathrm{Ca} /{ }^{12} \mathrm{C}$ ratio with injury in future studies. As such, here, we were not able to quantitatively detect ${ }_{95}$ increases in punctate ${ }^{40} \mathrm{Ca} /{ }^{12} \mathrm{C}$ signal in optic nerve vulnerable to secondary degeneration, consistent with release of $\mathrm{Ca}^{2+}$ from microdomains. It is important to note that there may have been an initial increase in Ca microdomain density in nerve vulnerable to secondary degeneration immediately following injury, which 100 declined to lower levels than normal by 5 minutes after injury. Further, it is possible that released $\mathrm{Ca}^{2+}$ may focus at the injury site, away from the area of secondary degeneration analyzed in the current study ${ }^{49}$. However, given that we have previously 
reported no difference in mean Ca signal between areas affected by the primary injury and those vulnerable to secondary degeneration ${ }^{28}$, it is possible that the total $\mathrm{Ca}^{2+}$ concentration actually falls throughout optic nerve following partial injury, 5 implying that the reported decrease in extracellular $\mathrm{Ca}^{2+}$ following spinal cord injury ${ }^{50}$ is not compensated for by intracellular $\mathrm{Ca}^{2+}$ concentration increases, as has been previously suggested ${ }^{51}$. Future studies will investigate these possibilities.

\section{Conclusions}

10 NanoSIMS analyses of Ca microdomains has provided novel insights regarding association with $\mathrm{P}$ and allowed quantitative, direct measures of complex $\mathrm{Ca}^{2+}$ microdomain changes in glial and axonal regions vulnerable to secondary degeneration following neurotrauma. The study has also illustrated the utility 15 of this technology, applicable to a wide range of mammalian systems in both normal and injured states.

\section{Notes and references}

${ }^{a}$ BioNano, School of Chemistry and Biochemistry, The University of Western Australia, 35 Stirling Hwy, Crawley WA 6009, Australia

$20{ }^{b}$ Experimental and Regenerative Neurosciences, School of Animal Biology, The University of Western Australia, 35 Stirling Hwy, Crawley WA 6009, Australia

${ }^{c}$ Centre for Microscopy, Characterisation and Analysis, The University of Western Australia, 35 Stirling Hwy, Crawley WA 6009, Australia

${ }_{25}$ *To whom correspondence should be addressed: Melinda Fitzgerald, Experimental and Regenerative Neurosciences, School of Animal Biology M317, The University of Western Australia, 35 Stirling Hwy, Crawley WA 6009, Australia. Tel: 6186488 2353, Fax: 6186488 7527, Email: lindy.fitzgerald@uwa.edu.au

30 1. S. Prilloff, M. I. Noblejas, V. Chedhomme and B. A. Sabel, Eur. J. Neurosci., 2007, 25, 3339-3346.

2. D. P. Stirling and P. K. Stys, Trends Mol. Med., 2010, 16, 160-170.

3. J. Knöferle, J. C. Koch, T. Ostendorf, U. Michel, V. Planchamp, P. Vutova, L. Tönges, C. Stadelmann, W. Brück, M. Bähr and P.

35 Lingor, Proc. Natl. Acad. Sci. U.S.A., 2010, 107, 6064-6069.

4. C. Giaume, F. Kirchhoff, C. Matute, A. Reichenbach and A. Verkhratsky, Cell Death Differ., 2007, 14, 1324-1335.

5. M. Fitzgerald, C. A. Bartlett, A. R. Harvey and S. A. Dunlop, J. Neurotrauma, 2010, 27, 439-452.

40 6. N. Hamilton, S. Vayro, F. Kirchhoff, A. Verkhratsky, J. Robbins, D. C. Gorecki and A. M. Butt, Glia, 2008, 56, 734-749.

7. T.-I. Peng and M.-J. Jou, Ann. N.Y. Acad. Sci., 2010, 1201, 183-188.

8. M. C. Camello-Almaraz, M. J. Pozo, M. P. Murphy and P. J. Camello, J. Cell. Physiol., 2006, 206, 487-494.

45 9. C. S. Lobsiger and D. W. Cleveland, Nat. Neurosci., 2007, 10, 13551360.

10. N. Hamilton, S. Vayro, R. Wigley and A. M. Butt, Glia, 2010, 58, 66-79.

11. R. Rizzuto and T. Pozzan, Physiol. Rev., 2006, 86, 369-408.

50 12. M. T. Alonso, C. Villalobos, P. Chamero, J. Alvarez and J. GarcíaSancho, Cell Calcium, 2006, 40, 513-525.

13. E. Shigetomi, S. Kracun, M. V. Sofroniew and B. S. Khakh, Nat. Neurosci., 2010, 13, 759-766.

14. M. J. Berridge, Cell Calcium, 2006, 40, 405-412.

55 15. E. Shigetomi, X. Tong, K. Y. Kwan, D. P. Corey and B. S. Khakh, Nat. Neurosci., 2012, 15, 70-80.

16. N. Kuga, T. Sasaki, Y. Takahara, N. Matsuki and Y. Ikegaya, J. Neurosci., 2011, 31, 2607-2614.

17. J. C. Koch, J. Knöferle, L. Tönges, U. Michel, M. Bähr and P.

$60 \quad$ Lingor, Nat. Protoc., 2011, 6, 1887-1896.

18. E. Shigetomi, E. A. Bushong, M. D. Haustein, X. Tong, O. JacksonWeaver, S. Kracun, J. Xu, M. V. Sofroniew, M. H. Ellisman and B. S. Khakh, J. Gen. Physiol., 2013, 141, 633-647.
19. M. A. Di Castro, J. Chuquet, N. Liaudet, K. Bhaukaurally, M. Santello, D. Bouvier, P. Tiret and A. Volterra, Nat. Neurosci., 2011, 14, 1276-1284.

20. S. D. Atkin, S. Patel, A. Kocharyan, L. A. Holtzclaw, S. H. Weerth, V. Schram, J. Pickel and J. T. Russell, J. Neurosci. Methods, 2009, 181, 212-226.

70 21. W. Sun, E. McConnell, J.-F. Pare, Q. Xu, M. Chen, W. Peng, D. Lovatt, X. Han, Y. Smith and M. Nedergaard, Science, 2013, 339, 197-200.

22. M. H. Myoga and W. G. Regehr, J. Neurosci., 2011, 31, 5235-5243.

23. C. Lechene, F. Hillion, G. McMahon, D. Benson, A. Kleinfeld, J. P.

75 Kampf, D. Distel, Y. Luyten, J. Bonventre, D. Hentschel, K. Park, S. Ito, M. Schwartz, G. Benichou and G. Slodzian, J.Biol., 2006, 5, 20.

24. R. Peteranderl and C. Lechene, J. Am. Soc. Mass Spectrom., 2004, 15, 478-485.

25. M. L. Steinhauser, A. P. Bailey, S. E. Senyo, C. Guillermier, T. S.

80 Perlstein, A. P. Gould, R. T. Lee and C. P. Lechene, Nature, 2012, 481, 516-519.

26. W. Song, H. Zukor, S.-H. Lin, A. Liberman, A. Tavitian, J. Mui, H. Vali, C. Fillebeen, K. Pantopoulos, T.-D. Wu, J.-L. Guerquin-Kern and H. M. Schipper, J. Neurochem., 2012, 123, 325-336.

85 27. L. E. Wedlock, M. R. Kilburn, J. B. Cliff, L. Filgueira, M. Saunders and S. J. Berners-Price, Metallomics, 2011, 3, 917-925.

28. J. Wells, M. R. Kilburn, J. A. Shaw, C. A. Bartlett, A. R. Harvey, S. A. Dunlop and M. Fitzgerald, J. Neurosci. Res., 2012, 90, 606-618.

29. M. Oheim, F. Kirchhoff and W. Stühmer, Cell Calcium, 2006, 40, $90 \quad 423-439$

30. M. Fitzgerald, C. A. Bartlett, L. Evill, J. Rodger, A. R. Harvey and S. A. Dunlop, Exp. Neurol., 2009, 216, 219-230.

31. M. Fitzgerald, S. C. Payne, C. A. Bartlett, L. Evill, A. R. Harvey and S. A. Dunlop, Invest. Ophthalmol. Vis. Sci., 2009, 50, 5456-5462.

95 32. J. Rauch, N. Volinsky, D. Romano and W. Kolch, Cell Commun. Signal., 2011, 9, 23.

33. K. M. Holmström, N. Marina, A. Y. Baev, N. W. Wood, A. V. Gourine and A. Y. Abramov, in Nat. Commun., Nature Publishing Group, a division of Macmillan Publishers Limited. All Rights Reserved., 2013, vol. 4, ch. January 15, 2013, p. 1362.

34. B. Liu, P. Wang, Z. Wang and W. Zhang, in PLoS One, Public Library of Science, 2011, vol. 6, ch. e16985, p. e16985.

35. J. Wang, J. Yang, P. Liu, X. Bi, C. Li and K. Zhu, Am. J. Pathol., 2012, 181, 746-752.

105 36. S. C. Payne, C. A. Bartlett, A. R. Harvey, S. A. Dunlop and M. Fitzgerald, Invest. Ophthalmol. Vis. Sci., 2012, 53, 6093-6101.

37. K. H. Lau, M. Christlieb, M. Schröder, H. Sheldon, A. L. Harris and C. R. M. Grovenor, J. Microsc., 2010, 240, 21-31.

38. S. E. Senyo, M. L. Steinhauser, C. L. Pizzimenti, V. K. Yang, L. Cai, 110 M. Wang, T.-D. Wu, J.-L. Guerquin-Kern, C. P. Lechene and R. T. Lee, Nature, 2013, 493, 433-436.

39. D. Sun and T. C. Jakobs, The Neuroscientist, 2012, 18, 567-588.

40. S. M. Davidson and M. R. Duchen, Cell Calcium, 2006, 40, 561-574.

41. P. L. Clode and A. T. Marshall, The Biological Bulletin, 2003, 204, 138-145.

42. R. Rizzuto, P. Pinton, W. Carrington, F. S. Fay, K. E. Fogarty, L. M. Lifshitz, R. A. Tuft and T. Pozzan, Science, 1998, 280, 1763-1766.

43. S. L. Budd and D. G. Nicholls, J. Neurochem., 1996, 67, 2282-2291.

44. J. A. Goldberg, J. N. Guzman, C. M. Estep, E. Ilijic, J. Kondapalli, J.

120 Sanchez-Padilla and D. J. Surmeier, Nat. Neurosci., 2012, 15, 14141421.

45. A. J. Kowaltowski, N. C. de Souza-Pinto, R. F. Castilho and A. E. Vercesi, Free Radic. Biol. Med., 2009, 47, 333-343.

46. K. Acsai, G. Antoons, L. Livshitz, Y. Rudy and K. R. Sipido, J. Physiol., 2011, 589, 2569-2583.

47. J. B. Gelderd and H. W. Sampson, Acta Neurobiol. Exp. (Wars.), 1990, 50, 601-608.

48. R. D. Happel, K. P. Smith, N. L. Banik, J. M. Powers, E. L. Hogan and J. D. Balentine, Brain Res., 1981, 211, 476-479.

130 49. W. Young and I. Koreh, Brain Res., 1986, 365, 42-53.

50. T. Moriya, A. Z. Hassan, W. Young and M. Chesler, J. Neurotrauma, 1994, 11, 255-263.

51. O. Honmou and W. Young, in The axon: structure function and pathophysiology, eds. S. G. Waxman, J. D. Kocsis and P. K. Stys, 
Oxford University Press, Oxford, United Kingdom of Great Britain and Northern Ireland, 1995, pp. 480-503.

52. P. L. Clode and A. T. Marshall, Protoplasma, 2003, 220, 153-161.

\section{Acknowledgements}

This work was supported by the Neurotrauma Research Program (Western Australia) and NH\&MRC (Grant ID: APP1028681). Prof. Dunlop is a Principal Research Fellow of the NH\&MRC, Australia (Grant ID: APP1002347). We thank Associate

${ }_{10}$ Professor Nathan J. Pavlos, The University of Western Australia, for kindly providing us with a sample of VDAC2 antibody; $\mathrm{Mr}$ Guy Ben-Ary of Cell Central, The University of Western Australia, for assistance with fluorescent microscopy; Mr John Murphy, The University of Western Australia, for assistance with 15 confocal microscopy and Associate Professor Jenny Rodger for critical review of the manuscript. The authors acknowledge the facilities, scientific and technical assistance of the Australian Microscopy and Microanalysis Research Facility at the Centre for Microscopy, Characterisation and Analysis, The University of

20 Western Australia, a facility funded by the University, State and Commonwealth Governments. This work was made possible in part by the OpenMIMS software whose development is funded by the NIH/NIBIB National Resource for Imaging Mass Spectrometry, NIH/NIBIB 5P41 EB001974-10. 